\title{
A FAST RECURSIVE ALGORITHM FOR COMPUTING CR-TYPE BOUNDS FOR IMAGE RECONSTRUCTION PROBLEMS
}

\author{
A.O. Hero*, J. A. Fessler**, W.L. Rogers** \\ *Dept. of Electrical Engineering and Computer Science and ${ }^{* *}$ Division of Nuclear Medicine \\ The University of Michigan, Ann Arbor, MI 48109
}

\begin{abstract}
In this paper we describe a method for computing matrix CR bounds for image reconstruction problems using an iterative algorithm that avoids the intractable inversion of the Fisher matrix required by direct methods. The algorithm produces a close approximation to the CR bound requiring only $O\left(n^{2}\right)$ floating point operations per pixel of interest; an order of magnitude savings relative to the $O\left(n^{3}\right)$ flops required by non-iterative methods. To illustrate the utility of our iterative algorithm we study a prototypical application: the dependence of achievable reconstruction accuracy on angular and radial sampling.
\end{abstract}

\section{INTRODUCTION}

The matrix CR bound on the covariance of any unbiased image reconstruction algorithm would be very useful for establishing fundamental limits on performance for image reconstruction and image parameter estimation. The unbiased CR bound matrix also enters into the bounds for biased estimators we are currently investigating. However, direct computation of the CR bound is difficult for image reconstruction problems since inversion of a very large dimensional $n \times n$ Fisher information matrix $\mathbf{F}_{Y}$ is required, where $n=m^{2}$ and image size is $m \times m$ pixels. For example, the CR bound for reconstruction of a small $q \times q$ ROI requires the corresponding $p \times p$ submatrix of the inverse of $\mathbf{F}_{Y}$, where $p=q^{2}$. The method of sequential partitioning [1] for computing the upper left $p \times p$ submatrix of $F_{Y}^{-1}$ and Cholesky based Gaussian elimination techniques [2] for computing the $p$ first columns of $F_{Y}^{-1}$ are efficient direct methods for obtaining the CR bound but require $O\left(n^{3}\right)$ floating point operations (flops). Even for a moderate image size, $O\left(n^{3}\right)$ flops is a tremendous computational load. For example, if the image is $128 \times 128$ then computing the inverse Fisher information matrix would require on the order of $4 \times 10^{12}$ (4 Tera) flops. For comparison, this number corresponds to a factor of $n=16,000$ more than the number of flops required for one iteration of the ML-EM image reconstruction algorithm!

\footnotetext{
${ }^{1}$ This research was supported in part by the National Science Foundation under grant BCS-9024370, the National Cancer Institute under grant R01-CA-54362-02, and DOE Alexander Hollaender Postdoctoral Fellowship.
}

0-7803-0883-2/93/\$3.00 @1993 IEEE
In this paper we give an iterative algorithm for approximating the CR bound. The method does not require inverting the Fisher information matrix and requires only $O\left(n^{2}\right)$ flops/iteration. The algorithm iteratively generates a sequence of approximation matrices which converges with exponential convergence rate to the actual CR bound matrix $\mathbf{F}_{Y}^{-1}$. The key to the algorithm is the specification of a diagonal "splitting" matrix $F$ which has the properties: 1) $\mathbf{F}^{-1}$ can be simply computed; 2) the eigenvalues of $\mathbf{I}-\mathbf{F}^{-1} \mathbf{F}_{\boldsymbol{Y}}$ are strictly between -1 and +1 . If these eigenvalues are nonnegative then the algorithm gives a sequence of approximations that are actually lower bounds which converge monotonically to the CR bound. To illustrate the utility of our iterative algorithm we study the impact of radial and angular sampling on achievable reconstruction accuracy for ECT.

\section{ITERATIVE CR BOUND ALGORITHM}

Let $\underline{\lambda}$ be the $n=m^{2}$ element vector of pixel intensities for an $m \times m$ image, $Y$ be a d-vector of projections data, and $A$ be the $d \times n$ system matrix associated with the tomographic system. Let $\underline{\theta}=G\left(\underline{\lambda}^{R O I}\right)$ be a set of parameters of interest which depend only on the pixel intensities $\underline{\lambda}^{R O I}$ within a region of interest (ROI) of the image, e.g. when reconstruction of the ROI is of interest $G(\underline{\lambda})=\underline{\lambda}^{R O I}$. Without loss in generality assume that the pixels have been ordered such that $\underline{\lambda}^{R O I}$ are the first $p$ elements of $\underline{\lambda}$. Then the CR bound on the covariance of any unbiased estimator $\underline{\hat{\theta}}$ of $\underline{\theta}$ is [5]:

$$
\operatorname{cov}_{\underline{\lambda}}(\underline{\hat{\theta}}) \geq \nabla_{\lambda} G \cdot \mathbf{F}_{\boldsymbol{Y}}^{-1} \cdot \nabla_{\lambda}^{T} G,
$$

where $\nabla_{\lambda} G$ is the gradient $\left[\partial G / \partial \lambda_{1}, \ldots, \partial G / \partial \lambda_{n}\right]$ of the function $G$ with respect to $\underline{\lambda}$, and $F_{Y}$ is the $n \times n$ Fisher information matrix of $\underline{\lambda}$ associated with $\underline{Y}$. Consider the partitions:

$$
F_{Y}=\left[\begin{array}{ll}
F_{11} & F_{21}^{T} \\
F_{21} & F_{22}
\end{array}\right] \quad F_{Y}^{-1}=\left[\begin{array}{cc}
D & C^{T} \\
C & E
\end{array}\right]
$$

where $\mathbf{F}_{11}, \mathbf{D}$ are $p \times p, \mathbf{F}_{21}, \mathbf{C}$ are $(n-p) \times p$, and $\mathbf{F}_{22}, \mathbf{E}$ are $(n-p) \times(n-p)$. Then since the last $n-p$ columns of $\nabla_{\lambda} G$ are zero the right hand side of the CR bound (1) becomes:

$$
\nabla_{\lambda} G \cdot \mathbf{F}_{Y}^{-1} \cdot \nabla_{\lambda}^{T} G,=\nabla_{\lambda^{R O I}} G \cdot \mathbf{D} \cdot \nabla_{\lambda \text { ROI }}^{T} G
$$


Note that the CR bound (1) only depends on $F_{Y}^{-1}$ through its $p \times p$ submatrix $D$. The submatrix $D$ can be found from either the first $p$ columns $\left[D, C^{T}\right]^{T}$ of the inverse $\mathbf{F}_{\boldsymbol{Y}}^{-1}$ or from the partitioned matrix inverse identity $\mathbf{D}=$ $\left[F_{11}-F_{12} F_{22}^{-1} F_{21}\right]^{-1}$; in both cases requiring $O\left(n^{3}\right)$ flops. Our recursive algorithm for approximating $\left[D, C^{T}\right]^{T}$ is based on finding a matrix $F$ that dominates $F_{Y}$ in the sense $\mathbf{F}-\mathbf{F}_{\boldsymbol{Y}} \geq \mathbf{0}$. This matrix is called a splitting matrix in analogy with classical methods of matrix computations [2]. It can be shown that this dominance condition guarantees that the eigenvalues of the matrix $I-F^{-1} \mathbf{F}_{Y}$ all lie in the interval $[0,1)[3]$. Define the relaxation parameter $0 \leq r \leq$ 1 and assume that all eigenvalues of $r F-F_{Y}$ lie in the interval $[-1,1]$. Apply the matrix form of the geometric series:

$$
\begin{aligned}
\mathbf{F}_{Y}^{-1} & =\left[r F-\left(r F-F_{Y}\right)\right]^{-1} \\
& =\frac{1}{r}\left[I-\left(I-\frac{1}{r} F^{-1} F_{Y}\right)\right]^{-1} \mathbf{F}^{-1} \\
& =\frac{1}{r}\left(\sum_{k=0}^{\infty}\left[I-\frac{1}{r} F^{-1} \mathbf{F}_{Y}\right]^{k}\right) \mathbf{F}^{-1}
\end{aligned}
$$

Next consider the truncated series for $l>0: \mathbf{B}^{(I)}=$ $\frac{1}{r}\left(\sum_{k=0}^{l}\left[\mathrm{I}-\frac{1}{r} \mathbf{F}^{-1} \mathrm{~F}_{Y}\right]^{k}\right) \mathrm{F}^{-1}$. Using induction on $k$ it is straightforward to verify that $\mathbf{B}^{(l)}$ can be obtained by iterating the following recursion out to $k=l$ :

$$
\mathbf{B}^{(k+1)}=\left[\mathbf{I}-\frac{1}{r} \boldsymbol{F}^{-1} \mathbf{F}_{Y}\right] \mathbf{B}^{(k)}-\frac{1}{r} \mathbf{F}^{-1}
$$

with initial condition $\mathbf{B}^{(0)}=0$, a $p \times p$ matrix of zeros. By right multiplying each side of the equality (2) by the matrix $\mathbf{E}=\left[\underline{e}_{1}, \ldots, \underline{e}_{p}\right]$, where $\underline{e}_{j}$ is the $j$-th unit vector in $\mathbb{R}^{n}$ we obtain the following recursion for the first $p$ columns $\mathbf{B}^{(k)} \mathbf{E}=\left[\underline{b}_{1}^{k}, \ldots, \underline{b}_{p}^{k}\right]$ of $\mathbf{B}^{(\mathfrak{l})}$ :

\section{Recursive Algorithm}

1. Initialization:

(a) $\beta^{(0)}=O$ an $n \times p$ matrix of zeros

(b) $\mathbf{M}=\left[\mathbf{I}-\frac{1}{r} \mathbf{F}^{-1} \mathbf{F}_{Y}\right]$ an $n \times n$ matrix

2. Recursion: For $k=0,1, \ldots, l$ :

$$
\beta^{(k+1)}=\mathbf{M} \cdot \beta^{(k)}+\frac{1}{r} \mathbf{F}^{-1} \mathbf{E}
$$

3. Bound on $\operatorname{cov}\left(\underline{\hat{\theta}}^{I}\right)$ :

$$
\mathbf{D}^{(l)}=\mathbf{E}^{T} \beta^{(l)} .
$$

The recursive algorithm yields an approximation $\mathbf{D}^{(l)}$ which converges to the $p \times p$ submatrix $\mathbf{D}$ of $\mathbf{F}_{\mathbf{Y}}^{-1}$ as $l \rightarrow \infty$. The rate of convergence is linear with convergence speed inversely proportional to the spectral radius $\rho(\mathbf{M})$, defined as the maximum magnitude eigenvalue of $\mathbf{M}$. Note that for $r=1$, since in this case $\mathbf{D}^{(l+1)}-\mathbf{D}^{(l)}=\mathbf{E}^{T} \cdot[\mathbf{I}-$ $\left.\frac{1}{r} \mathbf{F}^{-1} \mathbf{F}_{Y}\right]^{1+1} \mathbf{F}^{-1} \cdot \mathbf{E}$ is nonnegative definite, the recursive algorithm converges monotonically to $D$ as $l \rightarrow \infty$. Thus when $r=1$, for each $k>0 \nabla_{\lambda^{\text {nOI }}} G \cdot \mathbf{D}^{(k)} \cdot \nabla_{\lambda^{\text {ROI I }}}$ is a valid lower bound on $\operatorname{cov}(\underline{\hat{\theta}})$. By letting $r<1$ we can sacrifice monotone convergence to obtain an acceleration in the convergence rate.

When $p$ is much smaller than $n$, the $n \times n$ times $n \times p$ matrix multiplication $M \cdot \beta^{(k)}$ requires only $O\left(n^{2}\right)$ flops. Hence, in these cases the recursion (3) requires only $O\left(n^{2}\right)$ flops per iteration. Furthermore the initialization step of the recursion requires only $O\left(n^{2}\right)$ flops since $F$ is diago$\mathrm{nal}$. Therefore, assuming that the convergence rate $\rho(M)$ is sufficiently fast, the recursive algorithm can be used to compute an accurate approximation to the CR bound with an order of magnitude fewer flops than the direct Fisher inversion methods.

\section{SAMPLING STUDY}

The recursive algorithm of the previous section allows one to examine submatrices of CR bounds for ECT problems that would have been intractable by conventional methods. Define $A_{i, 0}$ and $\underline{A}_{0, b}$ the $i$-th row and $b$-th column, respectively, of the $d \times n$ system matrix $\mathbf{A}=\left(\left(A_{i, b}\right)\right)$. Under the assumption of Poisson emissions the general form for the $n \times n$ Fisher information matrix for ECT image $(\underline{\lambda})$ reconstruction is [3]:

$$
\mathbf{F}_{\mathbf{Y}}=\sum_{i=1}^{d} \frac{1}{\underline{\lambda}^{T} \underline{A}_{i, \bullet}} \underline{A}_{i, \bullet} \underline{A}_{i, \bullet}^{T}
$$

For this paper we used the following diagonal splitting matrix $\mathbf{F}$

$$
\mathbf{F}=\operatorname{diag}_{b}\left(\underline{1}^{T} \underline{A}_{, b}\right) \cdot \operatorname{diag}_{b}\left(\frac{1}{\lambda_{b}}\right)
$$

It can be shown [3] that $\mathbf{F}$ is the Fisher information matrix associated with the standard choice of complete data in the EM-ML algorithm: the set of pixel counts detected in the each of $d$ detector "tubes." In [3] it is also shown that, due to the data processing inequality for Fisher matrices, the Fisher information $\mathbf{F}$ for the complete data set dominates the Fisher information $\mathbf{F}_{Y}$ for the incomplete data set $\underline{Y}$.

To illustrate our recursive algorithm we investigated the effect of radial and angular sampling on the CR bound. In this study the forward projector corresponds to strip integrals whose widths equal the radial sampling interval. Three disk objects were studied, each with radii 13 pixels within a 32 by 32 image grid. The intensities in Disk 1 were all set to 1 , those in Disk 2 were drawn from a uniform distribution on $[1 / 2,3 / 2]$, and those in Disk 3 were drawn from a uniform distribution on $[0,1]$. The ROI consisted of a single pixel at the center of the disks which was set to 1 in all three cases. For each disk object, the CR bound on the variance of the center pixel was computed via 
the recursive algorithm for several different angular sampling intervals and different radial sampling intervals. The mean total number of detected events was held constant in all cases. We ran 1000 iterations of the recursive CR bound algorithm, and plotted bound versus iteration to see if convergence had occurred; it occurred in all but the most undersampled case.

It was observed that the CR bound results for uniform disk (Disk 1) and the low variation disk (Disk 2) agree to within $2 \%$. This is probably due to the insensitivity of the Fisher matrix (5) to object variation as long as the projections $\mathbf{A} \underline{\underline{\lambda}}$ do not vary. Table I displays the relative $\mathrm{CR}$ bounds for Disk 2, as a function of sampling, and Table II displays the results for Disk 3. "Bin Size" in these tables refers to the ratio of the radial sampling width to pixel width. We make the following observations based on Tables I and II. The fact that the bounds converged to a finite value is proof that the systems considered were of full rank. As expected, in all cases as radial or angular sampling improved, the CR bound decreased, reflecting the improved condition number of the system matrix. Also, all cases showed diminishing returns: beyond some point, further sampling is of little value. For Disks 1 and 2, it appears that no amount of angular sampling can make up for inadequate radial sampling. This can be seen from the fact that when the radial sampling is 0.5 times the pixel size, then as the angular sampling increases, the CR bound approaches an asymptote which is about $14 \%$ higher than the bound for 0.125 radial sampling. This implies that along the asymptote there is collinearity among the angular samples. However, this characteristic is object dependent; for Disk 3, the difference between 0.5 and 0.25 radial sampling was less than $4 \%$.

Huesman investigated the effects of radial and angular sampling [4] using an analytical approach that relied on the assumptions 1) that the noise variance is equal in all pixels, and 2) that the system matrix $A$ can be represented by line integrals. The iterative method we have described can be applied to any system model or noise distribution. The results in Tables I and II would suggest finer sampling is required than is typically implemented. This is due to the restriction of the ordinary CR bound to unbiased estimators. In most cases one may be willing to tolerate a small amount of bias in an image reconstruction algorithm in exchange for reduced variance. Although space limitations prevent us from going into details, we are currently investigating an extension of our CR bound methods to biased estimators, based on the uniform bound described in [6].

\begin{tabular}{|l|rrrrr|}
\cline { 2 - 6 } \multicolumn{1}{c|}{} & \multicolumn{5}{c|}{ Number of Angles } \\
\cline { 2 - 6 } \multicolumn{1}{c|}{} & 20 & 30 & 40 & 60 & 80 \\
\hline Bin Size & & & & & \\
\hline 1.0000 & 183.6 & 166.9 & 166.9 & 166.8 & 166.8 \\
0.5000 & 39.6 & 15.0 & 14.8 & 14.7 & 14.7 \\
0.2500 & 24.2 & 4.2 & 4.1 & 4.1 & 4.1 \\
0.1250 & 18.3 & 1.2 & 1.0 & 1.0 & 1.0 \\
0.0625 & 16.7 & 0.2 & 0.0 & 0.0 & 0 \\
\hline
\end{tabular}

Table I: \% increase in standard deviation relative to finest sampling for Disk 2.

\begin{tabular}{|l||rrrrr|}
\cline { 2 - 6 } \multicolumn{1}{c||}{} & \multicolumn{5}{c|}{ Number of Angles } \\
\cline { 2 - 6 } \multicolumn{1}{c|}{} & 20 & 30 & 40 & 60 & 80 \\
\hline Bin Size & & & & & \\
\hline 1 & 24.9 & 23.0 & 22.8 & 22.8 & 22.8 \\
0.5 & 17.8 & 4.9 & 3.8 & 3.8 & 3.8 \\
0.25 & 15.5 & 1.3 & 0.0 & 0.0 & 0 \\
\hline
\end{tabular}

Table II: \% increase in standard deviation relative to finest sampling for Disk 3.

\section{REFERENCES}

[1] A. R. Kuruc, "Lower bounds on multiple-source direction finding in the presence of direction-dependent antenna-array-calibration errors," Technical Report 799, M.I.T. Lincoln Laboratory, Oct., 1989.

[2] G. H. Golub and C. F. Van Loan, Matrix Computations (2nd Edition), The Johns Hopkins University Press, Baltimore, 1989.

[3] A. O. Hero and J. A. Fessler, "An iterative CR-bound algorithm and applications," Technical Report in prep., Comm. and Sig. Proc. Lab. (CSPL), Dept. EECS, University of Michigan, Ann Arbor, Nov 1992.

[4] R. H. Huesman, "The effects of a finite number of projection angles and finite lateral sampling of projections on the propagation of statistical errors in transverse section reconstruction," Physics in Medicine and Biology, vol. 22, no. 3, pp. 511-521, 1977.

[5] H. L. Van-Trees, Detection, Estimation, and Modulation Theory: Part I, Wiley, New York, 1968.

[6] A. O. Hero, "A Cramer-Rao Type Lower Bound for Essentially Unbiased Parameter Estimation", Tech. Rep. 890, MIT Lincoln Laboratory, January, 1992. 